\title{
Innovative Instrumentation for Analysis of Nanoparticles: The $\pi$ Steradian Detector
}

\author{
Nestor J. Zaluzec
}

Electron Microscopy Center, Materials Science Division, Argonne National Laboratory, Argonne, IL 60439

zaluzec@aaem.amc.anl.gov

\section{Aside}

The analysis was finally completed, the data graphed, and I had to admit to myself, it was looking like an auspicious moment. Only a few weeks earlier, Charlie Lyman and I had been discussing a project that I had been working on for some time, one in which I was admittedly being deliberately vague concerning the details. However, I had promised to keep him updated when it succeeded. Given the date (April 1st) and the data in hand, I couldn't resist sharing with him (and a few colleagues) the first results from that experiment-and my thinly veiled attempt at a quasi-April Fools Day joke. After we exchanged a number of emails that day, Charlie concluded his last message with the line that many of us have heard from both him and Ron Anderson: "A little more text and you can have an article in the July issue of MT." Have I gotten you curious? Well then read on!

\section{Introduction}

When studying the new generation of nano-materials, our ability to detect with high-collection efficiency and, in future, to analyze quantitatively smaller and smaller volumes of material has become an increasingly important part of our repertoire as microanalysts. Depending upon the specimen and the choice of probe, the nature of the signal we measure can vary over a wide range. Regardless of this variability, our proficiency in characterizing a material rests on our ability to detect a signal that is derived from the probe/specimen interaction. The better our resources (expertise, instrumentation, and data), the better our ability to undertake new and more complicated challenges.

In nano-materials research, one of the commonly employed tools is the electron microscope. After electrons, the signal most often measured in these instruments is that of emitted characteristic $\mathrm{x}$-rays. The $\mathrm{x}$-ray detector most used during the last forty years has been the energy-dispersive $\mathrm{x}$-ray spectrometer (EDS)-either the monolithic solid state $\mathrm{Si}(\mathrm{Li})$ detector or, more recently, the silicon drift detector (SDD) $[1,2]$. On one hand, these detectors are remarkably simple and efficient, but on the other hand, there are opportunities for improvement.

One of the factors governing our ability to measure an $\mathrm{x}$-ray signal is the detector geometrical collection efficiency, which is typically defined in terms of the collection solid angle $(\Omega)$ [3]. Figure 1 shows a calculation of the solid angle as a function of detector size and location for the standard detector geometry found in most electron-optical instruments. For EDS systems interfaced to scanning electron microscopes (SEM), values of $\Omega$ can range from $<0.005$ to $\sim 0.1$ steradians, whereas in transmission or scanning-transmission electron microscopes (TEM/STEMs) values of up to $\sim 0.1-0.2$ sr have become

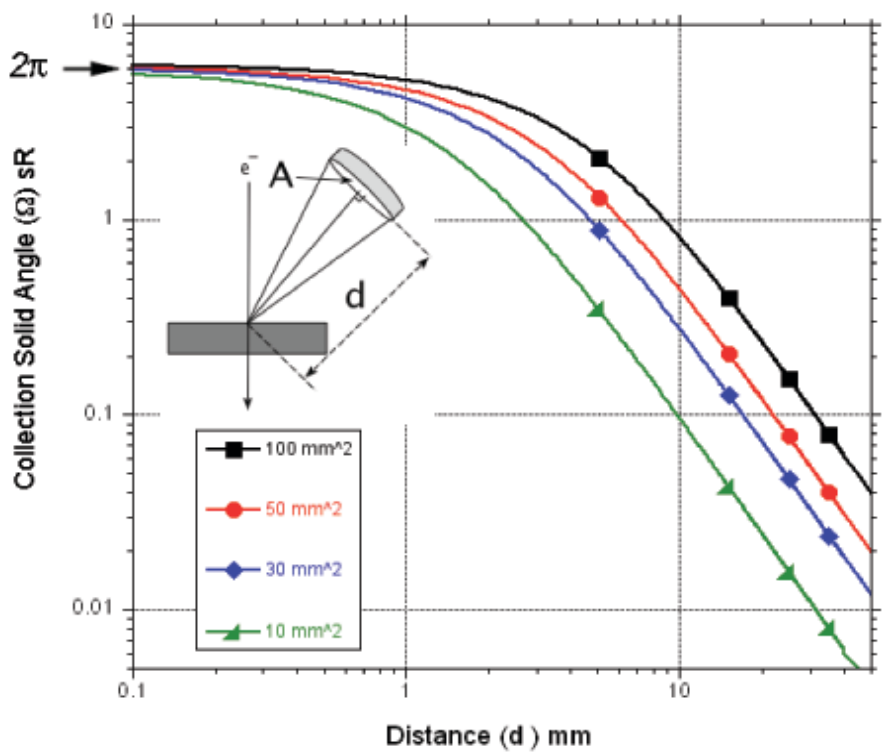

Figure 1: Collection solid angle $(\Omega)$ as a function of distance (d) for various detector active areas $\left(A=100,50,30\right.$, and $\left.10 \mathrm{~mm}^{2}\right)$. See reference [3] for calculation details.

routine. For the geometry illustrated in the inset of Figure 1, $100 \%$ collection efficiency corresponds to a solid angle of $2 \pi \mathrm{sr}$. Thus, a detector having a solid angle of $\sim 0.1$ sr collects only about $1.6 \%$ of the available signal. Said another way, for the majority of instruments we use today, we are neglecting more than $98 \%$ of the available signal. An improvement of nearly an order of magnitude is arguably a goal that should be readily achievable with a modicum of thoughtful and diligent effort. This was the objective of the research Charlie Lyman and I had been discussing.

\section{Collection Efficiency}

There are a number of approaches we can use to improve on the detector solid angle. For example, increasing the active area or the number of detectors has been employed in both SEM [4] and TEM/STEM [5] systems. When using quad-detector geometries, improvements of a factor of four have been reported [6]. However, to achieve an order of magnitude improvement, a radically different approach is needed.

To make a significant advance in $\Omega$, we first have to reconsider the nature of the measurement and the question posed. If the task is to measure the composition and structure of individual nano-particles, then it is essential to tailor the experimental configuration to maximize our ability to collect data. In research at ANL, nano-particles are typically dispersed on 


\section{SPI Supplies.}

\section{The complete source for all your microscopy needs...}

\section{just a click away. www.2spi.com}

Visit SPI Supplies to view the complete on-line catalog with up-to-the-minute product and pricing information.

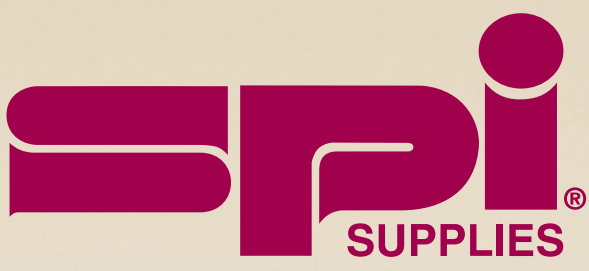

SPI Supplies Division of STRUCTURE PROBE, Inc.

P.0. Box $656 \bullet$ West Chester, PA 19381-0656 USA

Phone: 1-610-436-5400 • 1-800-2424-SPI (USA and Canada) • Fax: 1-610-436-5755• E-mail: sales@2spi.com 
ultrathin self-supporting films, such as those used in the TEM/ STEM. These include holey carbon films, thin self-supporting substrates (C, $\mathrm{SiN}, \mathrm{SiO}$ films, etc.), and substrates created using any number of modern atomic-layer deposition processes. The problem during nano-particle $\mathrm{x}$-ray analysis is not too many counts, but rather that there is so little material present that the signal is meager at best. Under good conditions, with particle sizes less than $10 \mathrm{~nm}$, the full-spectrum $\mathrm{x}$-ray count rate is rarely more than a few hundred counts/sec.

Given that we have decided that electrons are the probe of choice for characterization, then there are only three basic parameters that we can adjust to increase the measured $\mathrm{x}$-ray signal: accelerating voltage, beam current, and detector efficiency. While there is a variation in $\mathrm{x}$-ray generation with beam energy, which favors lower voltage operation, factor-of-ten improvements are not realizable using this approach [7]. Increasing the beam current is accompanied by poorer spatial resolution (usually due to larger probe sizes) and, in some materials, beam-induced damage, both detrimental to nano-particles characterization. Given the relatively low collection efficiency of existing detector configurations, improvements in this area would be the obvious direction to pursue.

The geometry we have been using for years in analytical microscopes for $\mathrm{x}$-ray analysis of nano-particles is simply not optimal. We are typically measuring less than $1 \%$ of the available signal, and clearly there is significant room for improvement. Using existing off-the-shelf technology, we have been constrained for decades by the microscope, its specimen stage, and the sample. So, the logical solution is to revisit the problem and do things differently. Ask yourself the simple question, how can I maximize the solid angle? Simple, just place the detector as close as possible to the sample-but also do this without interfering with the electron beam. For example, very small detectors located very close to the beam target area are a potential solution for bulk specimens, as we are constrained to looking only at the electron entrance surface of the sample [3]. However, for nano-particles on TEM quality thin films, there is no such constraint. There is no reason why one could not redesign and

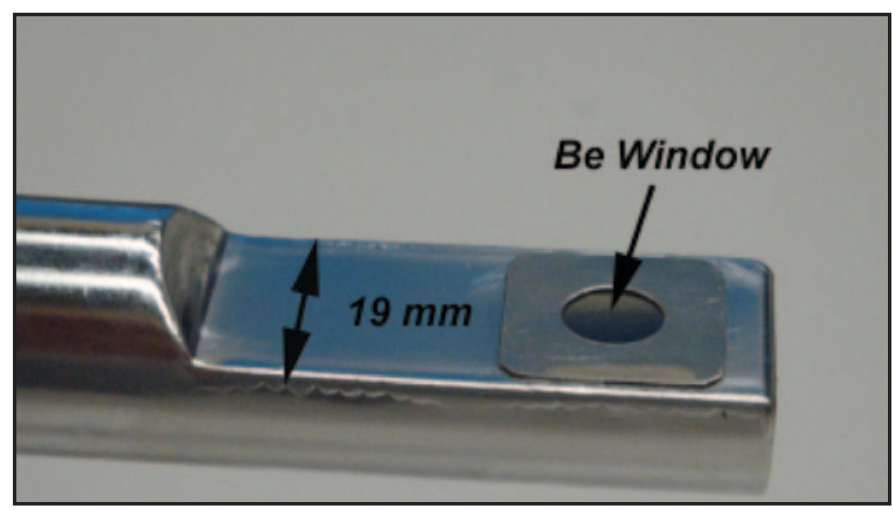

Figure 2: Prototype SDD detector in transmission geometry. The upward facing detector is enclosed within a $19 \mathrm{~mm}$ diameter stainless steel canister while the $42.5 \mathrm{~mm}^{2}$ crystal is protected by a $12.5 \mu \mathrm{m}$ thick beryllium window visible in the figure. The detector was custom manufactured by SII Nanotechnology USA Inc. relocate the $\mathrm{x}$-ray detector to a position where there is a lot of potential space, namely: beneath the nano-particle instead of above it.

To this end, a prototype $42.5 \mathrm{~mm}^{2}$ SDD $\mathrm{x}$-ray detector was built in collaboration with SII Nanotechnology USA (http:// www.siintusa.com). This new detector operates in a novel transmission configuration and has been initially interfaced to the column of an FEI Quanta 400 FEG-ESEM. The detector (Figure 2), having a nominal energy resolution of $130 \mathrm{eV} @ \mathrm{Mn}$ $\mathrm{Ka}$, is enclosed in a stainless steel housing (19 $\mathrm{mm}$ in diameter), whereas the active region of the SDD is protected from direct electron bombardment by a $12.5 \mu \mathrm{m}$ thick Be window. Experimental measurements confirm Kanaya-Okayama electron range calculations that electrons up to $25 \mathrm{kV}$ are stopped within this thickness and thus do not reach the active detector crystal. In this geometry, nano-particle specimens for analysis are supported on TEM grids held in a custombuilt, Be-shielded holder, which can be positioned using the existing SEM stage translation mechanisms. The SDD detector is inserted beneath the specimen by means of a standard linear insertion screw-drive, which is interfaced to a side entry port of the microscope. In this geometry, the SDD can be considered as a replacement for a STEM electron detector, with the caveat it is measuring the $\mathrm{x}$-ray signal instead of electrons. Accurate positioning of the specimen-to-detector distance is achieved by means of the z-height stepping motor of the stage, while imaging is accomplished using the conventional secondary and backscattered electron detectors. At closest approach, tilting of the specimen is limited at the present time, and correcting this requires a redesign of the specimen holder.

\section{Results}

The performance of this unorthodox solution to nano-particle analysis can be appreciated by reference to Figure

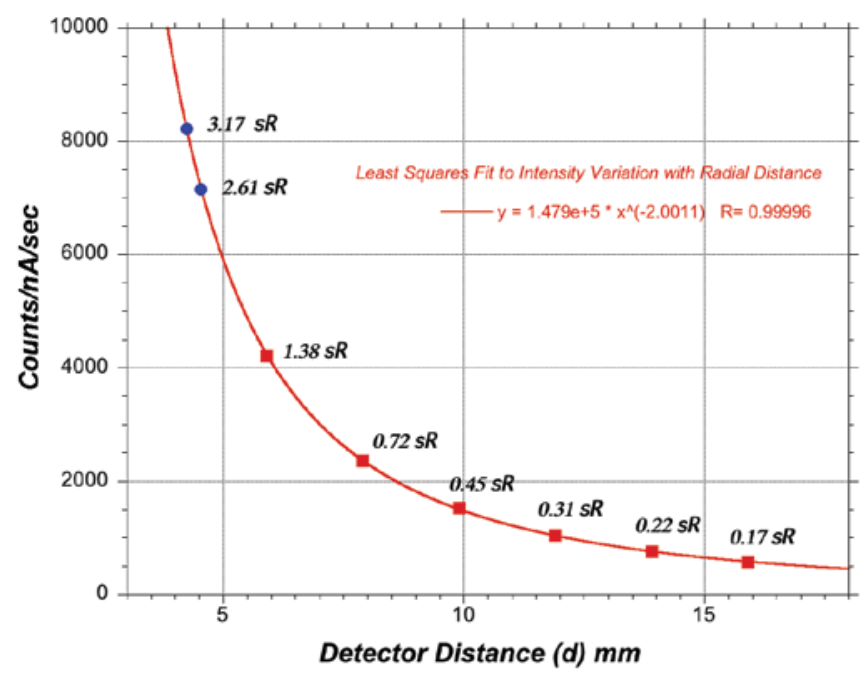

Figure 3: Experimentally measured $\mathrm{Au} L \alpha$ integrated x-ray peak intensity (data points) as a function of detector-specimen distance. Solid line is a least squares fit to the variation in the counts/nA/sec. The numerical values next to each data point $(3.17,2.61 \ldots .0 .17)$ represent the solid angle of the detector realized at each location. At the closest distance tested, the detector solid angle just exceeds $\pi \mathrm{sr}$. 


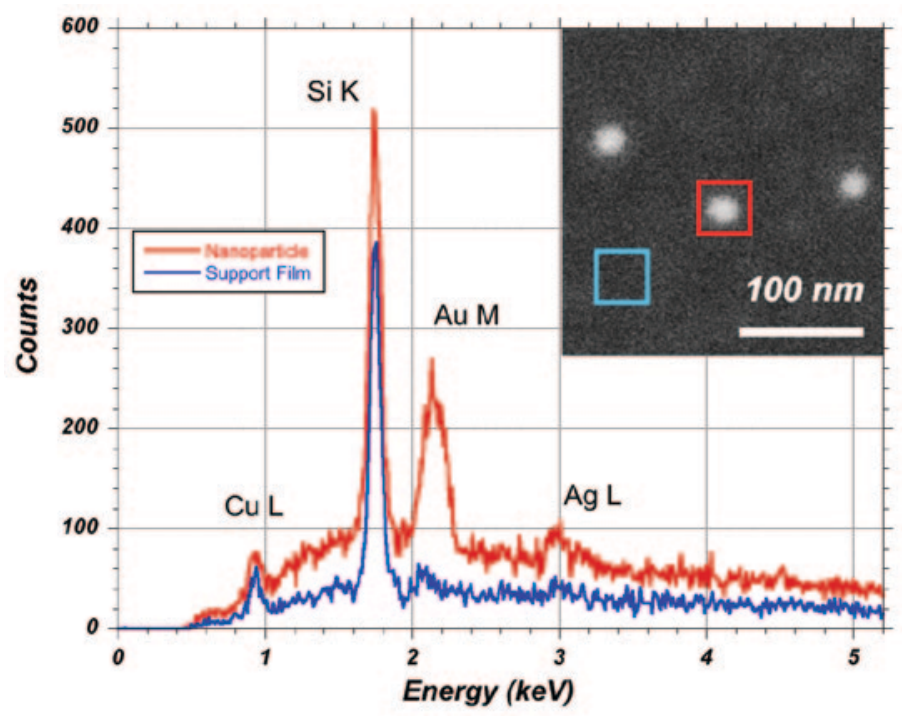

Figure 4: XEDS spectrum (red) obtained from a $20 \mathrm{~nm}$ diameter $\mathrm{Au}$ nano-particle (inset) compared to the background signal (blue) from the thin SiO support film. The integrated peak intensity of the $\mathrm{Au} \mathrm{M}$ lines is 2725 counts, acquisition time $=20$ live seconds, and the beam current $=0.025 \mathrm{nA}$ at $20 \mathrm{keV}$. The $\mathrm{Cu} \mathrm{L}$ and $\mathrm{Ag} \mathrm{L}$ lines are systems peaks.

3. Plotted here is the net $\mathrm{x}$-ray signal $(\mathrm{Au} \mathrm{La})$ measured as a function of specimen-to-detector distance, for a thin-film TEM test specimen of evaporated gold. The accelerating voltage was $20 \mathrm{kV}$, the spot size (SS) was \#2, and the beam current was $0.025 \mathrm{nA}$ using a $30-\mu \mathrm{m}$ objective aperture. These conditions correspond to the high-resolution imaging conditions in the SEM, rather than conventional microanalysis conditions (SS\#4, $0.8 \mathrm{nA}, 100 \mu \mathrm{m}$ aperture), and were specifically chosen to evaluate performance under conditions where high-resolution nano-particle imaging is conducted. The data points shown are the net counts/nA/sec of the integrated $\mathrm{Au}$ La peak intensity, recorded as a function of the distance (d) of the specimen from the EDS detector. In this transmission geometry a specimen-to-detector distance variation of $\sim 12 \mathrm{~mm}$ changes the $\mathrm{x}$-ray intensity by nearly $20 \mathrm{x}$, as documented in the data shown.

The solid line shown in Figure 3 is a linear least square fit of the data and demonstrates a $\mathrm{d}^{-2}$ dependence of the intensity with distance as is expected from first principles. Next to each data point is a numerical value that represents the solid angle achieved at each location. These values should be directly compared with the solid angles that are achieved in conventional geometries (Figure 1). At closest approach for this inaugural prototype, the maximum solid angle realized just exceeds $\pi \mathrm{sr}$, which represents collection of $50 \%$ of the available signal in this hemi-spherical geometry. This is more than a 30x improvement over the solid angle of conventional SEM geometries (typically $<0.1 \mathrm{sr}$ ) and exceeds the target goal of a ten-fold improvement over conventional detector orchestrations.

Figure 4 presents a spectrum obtained using this innovative technology from a real-world specimen consisting of a isolated 20-nm Au particle on a thin SiO film supported on a Cu TEM grid. The data were acquired from the indicated particle using a probe current of $0.025 \mathrm{nA}$ at $20 \mathrm{kV}$ for an acquisition live time of 20 seconds. The integrated intensity of the Au M peak contains 2725 counts and demonstrates that meaningful analysis of nano-particles can be readily achieved under these conditions. Also shown for comparison is the spectrum obtained from the $\mathrm{SiO}$ support film when the electron probe was translated off the particle. The $\mathrm{Cu}$ and $\mathrm{Ag} \mathrm{L}$ lines visible in the data are systems peaks from the TEM grid and the "silver paint" used to affix the grid to the holder.

\section{Discussion}

There are admittedly numerous problems yet to resolve and other geometries to address using this concept (i.e., TEM). For example, the generated bremsstrahlung created in a nano-particle by the incident electron probe will always be a present but should be only a small contribution due to the particle's small mass. However, as one can observe, there is significant background in the recorded spectra. This is generated by electron scattering elsewhere, and solutions have already been devised to mitigate this. Similarly, because of the transmission geometry, there is effectively no collimation for the detector; thus, characteristic system peaks $(\mathrm{Cu}, \mathrm{Fe}$, etc.) generated by scattering from various components of the instrument/system are observable. This difficulty is also being addressed. As one can appreciate, the results presented here represent only the initial stages of this $R \& D$ effort, which is time-consuming, not inexpensive, and often fraught with complications. Nevertheless, when it all comes together, the potential for improved $\mathrm{x}$-ray analysis, and the inherent positive effects on our science, is not far away.

So with a tip of my proverbial hat to Charlie for twisting my arm gently to write this synopsis, I'll leave you with the task of looking for a late-breaking poster at M\&M 2009 where a progress report on this system will be presented. See you in Richmond! VT

\section{References}

[1] R Fitzgerald, K Keil, and K F J Heinrich, Science 159 (1968) 528.

[2] G Bertuccio, A Castoldi, A Longoni, M Sampietro, and C Gauthier, Nucl Instr Meth, Phys Res A312 (1992) 613.

[3] N J Zaluzec, Microsc Microanal 15 (2009) 93-98.

[4] R Wuhrer and K Moran, Microsc Microanal 13 (suppl 2) (2007) 1350CD-1351CD.

[5] C E Lyman, J I Goldstein, D B Williams, D W Ackland, S von Harrach, A W Nicholls, and P J Statham, J Microsc 176 (1994) 85-98.

[6] P G Kotula, J R Michael, and M Rohde, Microsc Microanal 14 (suppl 2) (2008) 116.

[7] N J Zaluzec, Microsc Microanal 15 (suppl 2), in press (2009). [8] This work was supported by the US DoE, the Office of Science, at Argonne National Laboratory under Contract DE-AC02- 06CH11357. The SDD used for part of this work was custom built by SII Nanotechnology USA Inc. Support and encouragement from Dr. Valeri Saveliev, Director of R\&D at SII, is gratefully acknowledged. 